\title{
(C. COBENGE \\ COMPARATIVO ENTRE SIMULADORES APLICÁVEIS EM ENGENHARIA ELÉTRICA E AUTOMAÇÃO INDUSTRIAL VISANDO O ENSINO REMOTO
}

DOI: 10.37702/2175-957X.COBENGE.2021.3682

Camile Ribeiro Texca - camiletexca1@gmail.com

Instituto Federal do Paraná

Rua Claudir Kochinski 5

83602-636 - Campo Largo - PR

Eduarda de Matos Maria - eduardademmaria@gmail.com

Instituto Federal do Paraná

Rodovia Aníbal Khury 101

83650-000 - Balsa Nova - PR

Giovana Leal - gigiovanaleal26@gmail.com

Instituto Federal do Paraná

Ademar de Barros 126

83601-390 - Campo largo - PR

Beatriz dos Santos Pês - beatriz.santos@ifpr.edu.br

Instituto Federal do Paraná

Rua Monsenhor Ivo Zanlorenzi 1759

81210-000 - Curitiba - PR

Marcos Santos Hara - mshara@gmx.de

Instituto Federal do Paraná

Rua Herculano Carlos Franco de Souza 628

80240-290 - Curitiba - PR

Diego Tefili - diego.tefili@ifpr.edu.br

Instituto Federal do Paraná

Rua Dep Joaquim José Pedrosa 373

80025-120 - Curitiba - PR

Resumo: A pandemia de COVID-19 impôs uma série de dificuldades à sociedade. 
Dentre estas, a necessidade de ensinar e aprender de forma remota e emergencial. A modalidade de ensino remoto é especialmente desafiadora em cursos com alta carga tecnológica, nos quais as aulas experimentais em laboratório são fundamentais para a melhor compreensão dos conceitos. Na Engenharia Elétrica e suas ramificações, que demandam o entendimento de diversos fenômenos abstratos, os laboratórios são de particular importância. Simuladores têm grande potencial para auxiliar tanto docentes quanto discentes no processo de ensino-aprendizagem. No entanto, dada a grande gama de simuladores disponíveis, escolher um que se encaixe bem em cada situação pode não ser uma tarefa simples. Sendo assim, este trabalho propõe a elaboração de um quadro comparativo de simuladores disponíveis para disciplinas técnicas nas áreas de Engenharia Elétrica e Automação Industrial, elencando algumas de suas vantagens e desvantagens. Os simuladores foram listados e avaliados segundo uma série de critérios relacionados a custo, acessibilidade, plataforma de hardware necessária, usabilidade e praticidade. Foram avaliadas 28 componentes curriculares, para as quais foi possível sugerir 35 ferramentas de simulação. Os resultados obtidos até $o$ momento são apresentados na forma de tabelas que sintetizam características importantes e visam facilitar escolhas e aplicações por discentes, docentes e gestores de instituições de ensino.

Palavras-chave: Simuladores; Educação em Engenharia Elétrica; Ensino Remoto; Ferramentas Computacionais 


\section{COMPARATIVO ENTRE SIMULADORES APLICÁVEIS EM ENGENHARIA ELÉTRICA E AUTOMAÇÃO INDUSTRIAL VISANDO O ENSINO REMOTO}

\section{INTRODUÇÃO}

O novo coronavírus, Severe Acute Respiratory Syndrome Coronavirus 2 - SARSCov-2, que surgiu no final de 2019, impôs diversos desafios à sociedade como um todo. A pandemia que se seguiu atingiu os países com diferentes graus de intensidade, destacando a desigualdade nos mais diversos níveis (SÁ; SERPA, 2020). Apesar de o vírus ter levado aproximadamente 2 meses para atingir o Brasil, o país demonstrou não estar preparado para enfrentar a pandemia. Além das consequências para o sistema de saúde, há vários impactos sociais não tão óbvios que foram trazidos pelo vírus.

Os efeitos na educação, no que concerne a qualidade do aprendizado e o prejuízo ao desenvolvimento dos estudantes, são de particular gravidade e importância. Devido ao vírus, instituições de ensino viram-se obrigadas a adotar a modalidade remota de ensino, em uma tentativa de conter a propagação da doença. Esta adoção emergencial de uma modalidade de ensino para a qual grande parte das instituições não estavam preparadas, representa um obstáculo especialmente desafiador para os cursos nos quais os estudantes precisam ter aulas experimentais em laboratório, a fim de aprimorar seu aprendizado. Os cursos de Engenharia Elétrica e suas ramificações enquadram-se neste grupo. Os diversos fenômenos abstratos que precisam ser compreendidos pelos estudantes são mais facilmente assimilados com o uso de aulas práticas (LORETO-GÓMEZ et al., 2019).

Softwares de simulação ou simuladores são ferramentas baseadas em modelos de fenômenos e/ou sistemas reais que permitem ao usuário analisar estes fenômenos/sistemas sem necessariamente implementar uma montagem física. No que concerne à educação técnica e tecnológica, simuladores podem ser considerados ferramentas facilitadoras do processo de ensino-aprendizagem. Existem diversos estudos que apresentam as vantagens do uso de simuladores em cursos de nível técnico e superior na área de Engenharia Elétrica. Com base na literatura, pode-se afirmar que os simuladores auxiliam os estudantes a aprender e os professores a ensinar (LORETO-GÓMEZ et al., 2019). Uma vez que o acesso aos laboratórios esteja restringido, tais ferramentas deixam de serem complementos adicionais a serem utilizadas no processo de ensinoaprendizagem e ganham dimensão essencial, configurando-se em muitos casos como única alternativa para se aproximar dos benefícios providos pela experimentação prática.

Dada a atual situação de ensino remoto e o auxílio ao aprendizado fornecido pelos simuladores, tem-se que o uso destes torna-se, neste momento, especialmente recomendado e benéfico para o ensino de cursos na área de Engenharia Elétrica. Escolher um simulador adequado para cada caso, entretanto, pode não ser uma tarefa simples. Os docentes e discentes podem enfrentar dificuldades, devido à grande gama de simuladores disponíveis e à extensa variedade de características de cada software (JUUSO; SORSA, 2010). Sendo assim, este trabalho propõe a elaboração de um quadro comparativo de simuladores disponíveis para disciplinas técnicas nas áreas de Engenharia Elétrica e Automação Industrial, elencando algumas de suas vantagens e desvantagens. Os simuladores foram listados e avaliados segundo uma série de critérios relacionados a custo, acessibilidade, plataforma de hardware necessária, usabilidade e praticidade. $O$ escopo deste levantamento engloba disciplinas da área de Elétrica no curso superior de Engenharia Elétrica e nos cursos técnicos em Automação Industrial e Eletrotécnica do Instituto Federal do Paraná campus Campo Largo, mas supõe-se que possa ser facilmente adaptado para 
diferentes instituições. A finalidade principal é elaborar um banco de dados a ser divulgado para a comunidade acadêmica, disponibilizando informações sobre simuladores de forma concentrada, simples e acessível.

O presente trabalho está dividido da seguinte forma: a seção 2 reúne uma revisão bibliográfica de trabalhos que avaliaram como o uso de simuladores influencia o processo de ensino-aprendizagem, a seção 3 destina-se a apresentar a metodologia adotada durante o desenvolvimento do trabalho, a seção 4 apresenta os resultados já obtidos e, por fim, a seção 5 expõe as considerações finais e os desenvolvimentos futuros.

\section{REVISÃO BIBLIOGRÁFICA}

A formação na área de Engenharia Elétrica e suas ramificações deve fornecer aos estudantes conhecimento teórico e habilidades práticas para a solução de problemas. Para adquirir estas habilidades, é fundamental que o estudante passe por experiências em laboratórios didáticos. No entanto, no decorrer do curso, a carga horária que estudantes e professores podem dedicar a aulas experimentais costuma não ser suficiente para dar ao discente a segurança necessária para lidar com situações práticas. Embora esta deficiência possa ser suprida, por exemplo, com a oferta de projetos de pesquisa, dificilmente esta é uma solução que atinge todos os estudantes do curso. Uma forma de expor os estudantes a situações que podem ser encontradas no exercício da profissão é fazendo o uso de ferramentas computacionais capazes de simular essas situações (JUUSO; SORSA, 2010).

A difusão do uso de ferramentas computacionais, tais como os simuladores, nos cursos tecnológicos vinha sendo apontada como um dos aspectos caracterizadores do futuro da educação nas engenharias Elétrica e de Computação há alguns anos (BERRY; DIPIAZZA; SAUER, 2003). Historicamente há evidências de que o emprego de instrumentos que transcendam o método de ensino tradicional (exposições teóricas em sala de aula) é uma das melhores formas de atingir os objetivos de aprendizagem, alcançando o perfil do egresso desejado (FELDER et al., 2000; RUGARCIA et al., 2000).

Em 2019, Loreto-Gómez et al. desenvolveram uma pesquisa com o objetivo de comparar o desempenho acadêmico de dois grupos de estudantes de um curso de robótica. Um dos grupos assistiu a aulas teóricas tradicionais, enquanto o outro grupo, além das aulas tradicionais, foi submetido ao uso de modelos 3D e softwares de simulação. A avaliação final de desempenho demonstrou que os estudantes que fizeram uso de simuladores e modelos apresentaram melhores resultados, indicando que a compreensão dos conceitos necessários foi superior neste grupo, em comparação com os estudantes do primeiro grupo (LORETO-GÓMEZ et al., 2019).

O estudo desenvolvido por Loreto-Gómez et al. contribui para uma linha de pesquisa que conta com diversos trabalhos de pesquisadores de todo o mundo. De forma geral, estes estudos são divididos por áreas específicas e buscam avaliar o impacto do uso de simuladores no desempenho dos discentes. Dentre estes trabalhos, cita-se o estudo feito por Swain e Anderson, que avaliou o efeito da inserção de ferramentas de simulação em cursos de Microcontroladores e Organização Computacional a fim de melhorar o aprendizado dos estudantes (SWAIN; ANDERSON, 2008). A mesma avaliação foi conduzida por Campbell et al. considerando a área de Eletrônica (CAMPBELL et al., 2002), Alsadoon et al. estudaram o impacto positivo do uso de simuladores em um curso de Lógica Digital (ALSADOON; PRASAD; BEG, 2016) e Ayasun e Nwankpa, em um curso de Máquinas Elétricas (AYASUN; NWANKPA, 2005). Mesmo envolvendo áreas diferentes da Engenharia Elétrica, tais estudos evidenciaram que o uso de simuladores proporciona melhoria no processo ensino-aprendizagem. 
Em disciplinas que envolvem conhecimentos de Eletricidade, as ferramentas de simulação são particularmente úteis, pois mesmo as aulas experimentais não são capazes de fazer o estudante visualizar os fenômenos abstratos que precisam ser compreendidos. Os simuladores podem fazer uso de interfaces gráficas capazes de ilustrar o invisível, facilitando a compreensão dos estudantes (LORETO-GÓMEZ et al., 2019).

Dado o exposto, conclui-se que o uso de softwares de simulação como ferramenta de ensino, complementando as aulas teóricas e experimentais, tem o potencial de facilitar e ampliar o aprendizado dos estudantes, além de tornar o processo formativo mais interessante. No entanto, a depender do currículo do curso e da forma como o docente apresenta a ferramenta aos discentes, os resultados podem não ser tão positivos. Estudantes matriculados em cursos com currículos inflados podem encarar as atividades com os simuladores como uma sobrecarga (CAÑIZARES; FAUR, 1997). No estudo conduzido por Cañizares e Faur, mesmo discentes que reconheceram o valor agregado pelas ferramentas de simulação consideraram que as tarefas extra-classe os sobrecarregaram. Apesar de se tratar de um estudo que envolve ferramentas de simulação da década de 1990, que usualmente possuíam usabilidade limitada, a possibilidade de sobrecarregar os estudantes com o desenvolvimento de maior quantidade de tarefas deve ser considerada. Além disso, docentes pouco familiarizados com os softwares podem ser relutantes ao uso de simuladores nas disciplinas que conduzem.

A fim de difundir o uso de simuladores nos cursos de Engenharia Elétrica e Automação Industrial do IFPR campus Campo Largo, este trabalho propõe a elaboração de um quadro comparativo detalhado, avaliando algumas das ferramentas disponíveis de acordo com critérios preestabelecidos. Entende-se que a disponibilização de informação consolidada sobre os simuladores facilita e até mesmo incentiva o uso destes instrumentos, importantes como ferramentas educacionais e essenciais para o ensino remoto de componentes curriculares dos eixos de formação técnica. Para docentes e discentes, possuir dados concentrados e facilmente acessíveis, torna a tarefa de escolher um simulador e avaliar suas vantagens e desvantagens mais simples. Para as instituições de ensino, este quadro comparativo pode servir como fonte de pesquisa sobre softwares cuja licença se está considerando adquirir. Na próxima seção, a metodologia adotada para o desenvolvimento deste trabalho é apresentada.

\section{METODOLOGIA}

A concepção e elaboração deste trabalho deveu-se à percepção de uma oportunidade de melhoria, por meio do uso de ferramentas de simulação, nos métodos de ensino-aprendizagem dos cursos de Engenharia Elétrica e técnico em Automação Industrial do IFPR campus Campo Largo. Deste modo, o primeiro passo para alcançar os objetivos propostos consistiu em analisar os Projetos Pedagógicos dos Cursos, PPCs, a fim de levantar quais disciplinas das áreas técnicas poderiam ser beneficiadas pela inclusão de simuladores nos métodos de ensino. A elaboração de uma lista de simuladores passíveis de uso em cada uma destas disciplinas foi o segundo passo da pesquisa. Para fundamentar a escolha de uma ferramenta de simulação, o terceiro passo consistiu em estabelecer critérios iniciais de classificação. Estes critérios são relativos a custo, acessibilidade, plataforma de hardware necessária, usabilidade e praticidade. De posse de todos estes dados, prosseguiu-se para a elaboração de planilhas que apresentam as informações de forma consolidada e prática. Por haver diversas opções e critérios, foi definido que a apresentação visual dos resultados deveria proporcionar destaque através do uso de marcadores e cores. Os passos da metodologia serão detalhados nas subseções a seguir. 


\subsection{Levantamento das disciplinas por meio dos PPCs}

Os PPCs dos cursos de Engenharia Elétrica e técnico em Automação Industrial podem ser encontrados no site do IFPR campus Campo Largo. Atualmente, existem dois PPCs em vigência para o curso de Automação Industrial, um de 2016 e um de 2019. O levantamento das disciplinas considerou as grades curriculares constantes nos dois PPCs. Existem várias disciplinas com nomes similares nos cursos de Engenharia Elétrica e de Automação Industrial, e.g., Circuitos Elétricos. No entanto, o docente deve atentar-se para as diferenças inerentes entre cursos de nível superior e de nível médio, presentes nas ementas, no momento de escolher o simulador adequado.

\subsection{Lista de Simuladores}

Para sugerir simuladores em cada uma das disciplinas listadas na Tabela 1, foram necessárias pesquisas feitas online, consulta a professores de áreas específicas e discussões entre autores e colaboradores. Buscou-se sugerir softwares cujo uso se demonstrou mais difundido, uma vez que estas ferramentas apresentam uma comunidade de utilizadores que fornecem e trocam informações ativamente, resultando usualmente em documentação mais abrangente.

\subsection{Critérios de avaliação iniciais}

Conforme já mencionado, os critérios de classificação dos simuladores referem-se a custo, acessibilidade, plataforma de hardware necessária, usabilidade e praticidade. Para definir quais parâmetros objetivos poderiam ser utilizados para qualificar estes aspectos, foram consultados fóruns de discussão online e materiais disponibilizados pelos desenvolvedores das ferramentas de simulação. A experiência dos discentes e docentes que compõem o grupo de autores também foi considerada. Além disso, procurou-se utilizar parâmetros que caracterizem a acessibilidade e facilidade de uso, considerando as limitações impostas pelo ensino remoto.

Desta forma, chegou-se aos seguintes critérios classificatórios: (1) ser Open Source, (2) possuir aplicativo para Android, (3) possuir aplicativo para iOS, (4) possibilitar uso no browser, (5) ter sido atualizado nos últimos 2 anos, (6) possuir versão em língua portuguesa, (7) realizar simulações através de esquemáticos, (8) realizar simulações que emulam a montagem física, (9) número de disciplinas atendidas e (10) documentação disponível.

Um software Open Source, ou de código aberto, é concebido sobre o princípio de que o produto deve ser disponibilizado juntamente com seu código fonte. Existem diversas vantagens que acompanham esta concepção, dentre as principais está a possibilidade de indivíduos desenvolverem trabalhos transformadores a partir de uma ideia original (HERON; HANSON; RICKETTS, 2013).

Uma vez que este trabalho visa o estudo remoto, é necessário considerar as diversas condições sociais em que os estudantes podem estar inseridos. Há várias situações em que os aplicativos para smartphones tornam-se vantajosos. Eles permitem que estudantes que não possuem acesso a um computador ou notebook possam utilizar o simulador. Além disso, o estudante pode acessar e alterar facilmente seus projetos. A possibilidade de uso no browser objetiva facilitar o acesso ao uso de simuladores para estudantes que não possuem computadores que permitam a instalação de mais softwares devido a configurações de hardware ou eventuais limitações impostas pelo sistema operacional utilizado.

O critério relativo a atualizações nos últimos dois anos pretende mensurar a condição de desenvolvimento e de uso da ferramenta de simulação. Não possuir novas versões há 
mais de dois anos pode indicar que o simulador encontra-se defasado e não será mais continuado pelo desenvolvedor. Este parâmetro pode auxiliar na tomada de decisão por parte de gestores, quando se está considerando adquirir a licença de um software. O número de disciplinas atendidas é outra característica que auxilia nesta tomada de decisão.

Ferramentas de simulação que possuem versões em língua portuguesa tornam o acesso e uso mais democráticos. A documentação disponível também deve ser avaliada, quanto mais conhecimento e discussões existirem, maior é a probabilidade de que o estudante consiga exercer autonomia e protagonismo no processo formativo. Além disso, mais fácil será para o professor sanar quaisquer questões que surjam durante o uso da ferramenta. A forma da interface gráfica de simulação disponível (se por diagrama esquemático ou emulação da montagem física) consiste em uma informação que auxilia na escolha de uma ferramenta adequada ao objetivo que se busca alcançar com seu uso.

Além dos parâmetros mencionados, foi solicitado às estudantes que compõem o grupo de autores que apontassem duas características que elas consideravam particularmente interessantes em cada simulador. Apesar de subjetivos, estes apontamentos são proveitosos, pois buscam sumarizar pontos positivos ou negativos de cada simulador, segundo a percepção das estudantes.

Por fim, ressalta-se que estes critérios não devem ser vistos como eliminatórios. Eles consistem apenas em informação coligida com o objetivo de auxiliar no conhecimento das ferramentas e suas características. A próxima seção apresenta os resultados obtidos.

De maneira resumida, o fluxo da Figura 1, ilustra os principais passos da metodologia desenvolvida.

Figura 1 - Diagrama ilustrativo dos passos da metodologia.

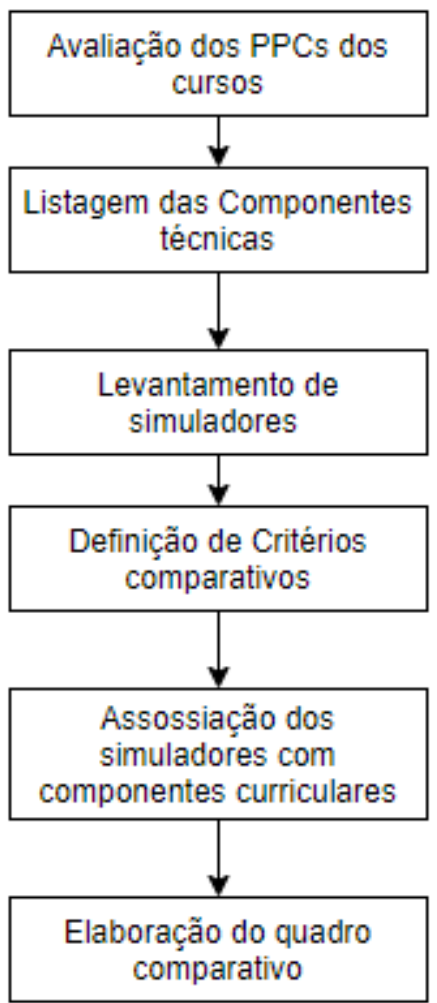

Fonte: elaborada pelos autores. 


\section{$4 \quad$ RESULTADOS}

No total, foram considerados 35 simuladores e 28 disciplinas diretamente ligadas com a área de concentração dos cursos. Disciplinas da base comum, como Cálculo e Física, que também podem ser beneficiadas pelo uso de simuladores, não foram consideradas neste levantamento. As componentes curriculares que foram relacionadas e que, portanto, terão ferramentas de simulação sugeridas estão listadas na Tabela 1.

Tabela 1 - Lista de componentes Curriculares levantadas por meio do PPC dos cursos de Engenharia Elétrica e técnico em Automação Industrial.

\begin{tabular}{|c|c|c|c|}
\hline Componente Curricular & E.E.* & Aut. $19 *$ & Aut. $16^{*}$ \\
\hline Introdução à Engenharia & $X$ & & \\
\hline Desenho Técnico & $X$ & & 0 \\
\hline Eletricidade Básica & $X$ & $\mathrm{x}$ & 0 \\
\hline Eletromagnetismo & $x$ & & \\
\hline Circuitos Elétricos I & $x$ & $x$ & 0 \\
\hline Circuitos Elétricos II & $X$ & & \\
\hline Máquinas Elétricas & $X$ & & 0 \\
\hline Programação I & $\mathrm{X}$ & $\mathrm{x}$ & 0 \\
\hline Programação II & $X$ & $x$ & \\
\hline Eletrônica Digital & $X$ & $x$ & 0 \\
\hline Microcontroladores & $X$ & $x$ & \\
\hline Lógica Programável & $X$ & & \\
\hline Eletrônica Analógica & $\mathrm{X}$ & $x$ & 0 \\
\hline Eletrônica de Potência & $X$ & & \\
\hline Sinais e Sistemas & $\mathrm{X}$ & & \\
\hline Amplificadores e Filtros & $X$ & & \\
\hline Acionamentos Elétricos e CLP & $X$ & $x$ & 0 \\
\hline Instalações Elétricas & $X$ & $\mathrm{x}$ & 0 \\
\hline Sistemas Supervisórios & $X$ & $x$ & 0 \\
\hline Redes Industriais & $X$ & $x$ & \\
\hline Robótica Industrial & $X$ & $x$ & \\
\hline Instrumentação Industrial & $X$ & $x$ & 0 \\
\hline Modelagem e Simulação & $X$ & & \\
\hline Controle Contínuo & $X$ & $x$ & 0 \\
\hline Modelagem de Sistemas Discretos & $X$ & & \\
\hline Controle Discreto & $X$ & & \\
\hline Técnicas de Controle Moderno & $X$ & & \\
\hline Tópicos em Inteligência Artificial & $x$ & & \\
\hline
\end{tabular}

A fim de apresentar os dados consolidados de uma maneira que facilite a extração da informação, as tabelas foram pensadas de forma visual. Na Figura 2, que apresenta a 
relação dos simuladores sugeridos e quais componentes curriculares eles atendem, as disciplinas foram ordenadas em grupos, de acordo com os conhecimentos estudados. A cada um destes grupos foi atribuída uma cor. Por exemplo, a cor verde claro foi atribuída para o grupo de disciplinas que usa conhecimentos da área de Eletricidade. Além disso, tanto na Figura 2 quanto na Figura 3, um esquema de cores diferencia as linhas relativas a softwares gratuitos, pagos com versão gratuita e pagos.

Figura 2 - Relação de simuladores e componentes curriculares.

\begin{tabular}{|c|c|c|c|c|c|c|c|c|c|c|c|c|c|c|c|c|c|c|c|c|c|c|c|c|c|c|c|c|c|}
\hline & & 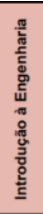 & 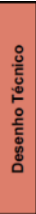 & 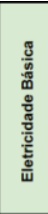 & 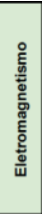 & 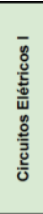 & 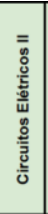 & 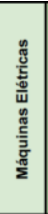 & 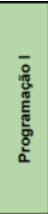 & 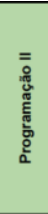 & 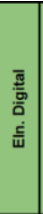 & 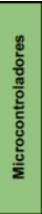 & 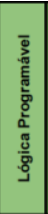 & $\begin{array}{l}\frac{\mathrm{g}}{\mathrm{g}} \\
\frac{\mathrm{g}}{\mathrm{g}} \\
\frac{\mathrm{e}}{4} \\
\frac{\dot{\mathrm{i}}}{\mathrm{w}}\end{array}$ & 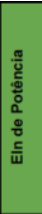 & 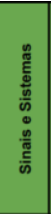 & 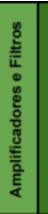 & 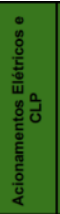 & 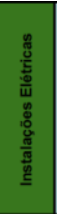 & 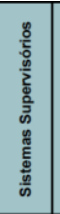 & 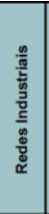 & 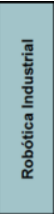 & 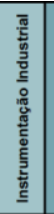 & 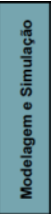 & 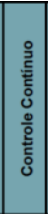 & 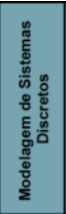 & 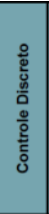 & 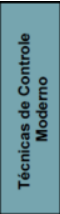 & 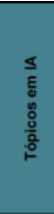 \\
\hline \multirow{23}{*}{ 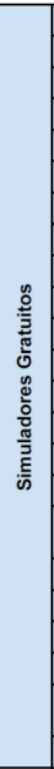 } & Tinkercad & $x$ & & $x$ & & $x$ & & & & & $x$ & $x$ & & $x$ & & & $x$ & 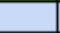 & & & & & $x$ & & $x$ & & & & \\
\hline & Falstad & & & & $x$ & $x$ & $x$ & & & & 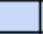 & & & $x$ & & $x$ & $x$ & & & & & & 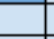 & 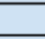 & $\square$ & & & & \\
\hline & Logisim & & & & & & & & & & $\mathrm{x}$ & & & & & & & & & & & & & & & & & & \\
\hline & CadeSimu & & & & & & & $x$ & & & & & & & & & & $x$ & & & & & & & & & & & \\
\hline & OMNET++ & & & & & & & & & & & & & & & & & & & & $x$ & & & & 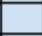 & & & & \\
\hline & $\mathrm{ns} \cdot 3$ & & & & & & & & & & & & & & & & & & & & $x$ & & & & & & & & \\
\hline & PICSimLab & & & & & & & & & & & $x$ & & & & & & & & & & & & & & & & & \\
\hline & AutoCad & & $x$ & & & & & & & & & & & & & & & & & & & & & & & & & & \\
\hline & \begin{tabular}{|l|} 
WEG CLIC02 \\
\end{tabular} & & & & & & & & & & & & & & & & & $x$ & & & & & & & & & & & \\
\hline & \begin{tabular}{|l|} 
MotorAnalysis \\
\end{tabular} & & & & & & & $x$ & & & & & & & & & & & & & & & & & & & & & \\
\hline & \begin{tabular}{|l|} 
ScadaBR \\
\end{tabular} & & & & & & & & & & & & & & & & & & & $x$ & & & & & 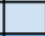 & & & & \\
\hline & JMCAD & & & & & & & & & & & & & & & & & & & & & $x$ & & $x$ & $x$ & & & & \\
\hline & QUCS & & & & & $x$ & $x$ & & & & & & & $x$ & & & & & & & & & & & & & & & \\
\hline & SimullDE & & & & & $x$ & $x$ & & & & & $x$ & & & 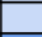 & & & & & & & & & & 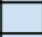 & & & & \\
\hline & NGSpice & & & & & $x$ & $\bar{x}$ & & & & & & & $x$ & $x$ & & & & & & & & & & & & & & \\
\hline & TimingEditor & & & & & & & & & & $x$ & & & & & & & & & & & & & & & & & & \\
\hline & $\begin{array}{c}\text { Digital Logic } \\
\text { Desing }\end{array}$ & & & & & & & & & & $x$ & & & & & & & & & & & & & & & & & & \\
\hline & GPSim & & & & & & & & & & & $x$ & & & & & & & & & & & & & & & & & \\
\hline & EKTS & & & & & & & & & & & & & & & & & $x$ & & & & & & & & & & & \\
\hline & \begin{tabular}{|l|} 
Delta ISPSoft \\
\end{tabular} & & & & & & & & & & & & & & & & & $x$ & & & & & & & & & & & \\
\hline & \begin{tabular}{|l|} 
Delta WPLSoft \\
\end{tabular} & & & & & & & & & & & & & & & & & $x$ & & & & & & & & & & & \\
\hline & \begin{tabular}{|l|} 
Electric VLSI \\
Design System
\end{tabular} & & & & & & & & & & & & $\mathrm{x}$ & & & & & & & & & & & & & & & & \\
\hline & \begin{tabular}{|l|} 
Circuit Lab \\
\end{tabular} & & & $\mathrm{x}$ & & $x$ & $x$ & & & & $x$ & & & $x$ & & & & & & & & & & & & & & & \\
\hline \multirow{5}{*}{ 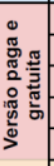 } & NanoCad & & $x$ & & & & & & & & & & & & & & & & $x$ & & & & & & & & & & \\
\hline & Matlab & $x$ & & & $x$ & & & & $x$ & $x$ & & $x$ & & 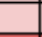 & & $x$ & 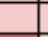 & & & & & $x$ & $x$ & $x$ & $x$ & $x$ & $x$ & $x$ & $x$ \\
\hline & Orcad & & & & & $x$ & $x$ & & & & & & & $x$ & $\bar{x}$ & & $x$ & & & & & & & & & & & & \\
\hline & EveryCircuit & & & $x$ & & $x$ & & & & & $x$ & & & $x$ & $x$ & & $x$ & & & & & & & & & & & & \\
\hline & Pycharm & $\bar{x}$ & & & $\bar{x}$ & & & & $\bar{x}$ & $\bar{x}$ & & $\bar{x}$ & & & & $\bar{x}$ & & & & & & & & $\bar{x}$ & & $\bar{x}$ & $\bar{x}$ & $\bar{x}$ & $\bar{x}$ \\
\hline \multirow{7}{*}{ 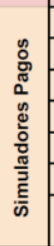 } & FRITIZING & $x$ & & & & $x$ & $x$ & & & & $x$ & & & & & & & & & & & & & & $\mathrm{x}$ & & & & \\
\hline & $\begin{array}{l}\text { Protheus } \\
\end{array}$ & & & & & $x$ & $x$ & & & & & $x$ & & $x$ & $x$ & & & & & & & & & & & & & & \\
\hline & AutoCad & & & & & & & & & & & & & & & & & & $x$ & & & & & & & & & & \\
\hline & Matlab & & & & & & & & & & & & & & & & & & & & & & & & & & & & \\
\hline & Simulavr & & & & & & & & & & & $x$ & & & & & & & & & & & & & & & & & \\
\hline & DraftSight & & $x$ & & & & & & & & & & & & & & & & & & & & & & & & & & \\
\hline & $\begin{array}{c}\text { Automation } \\
\text { Studio }\end{array}$ & & & & & & & $x$ & & & & & & & & & & & & & & $x$ & & & & & & & \\
\hline
\end{tabular}

Fonte: elaborada pelos autores.

A Figura 3 apresenta a classificação dos simuladores segundo os critérios considerados, explicados na seção 3.3. 
Figura 3 - Classificação dos simuladores sugeridos conforme os critérios estabelecidos (seção 3.3).

\begin{tabular}{|c|c|c|c|c|c|c|c|c|c|c|c|c|c|}
\hline & & 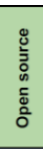 & 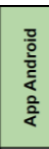 & 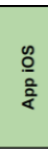 & 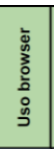 & 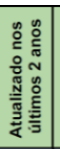 & 递 & 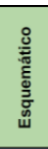 & 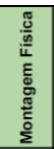 & 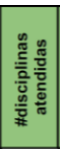 & 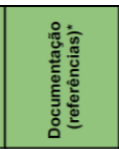 & 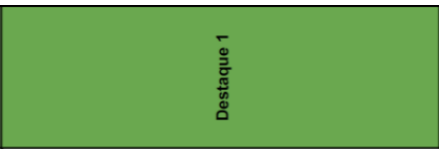 & 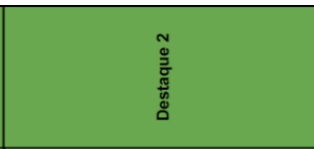 \\
\hline & Tinkercad & $x$ & & & $x$ & $\mathrm{x}$ & $x$ & $x$ & $x$ & 9 & Excelente & Interface que emula montagens em bancada. & Grande variedade de exemplos. \\
\hline & Falstad & & & & $\mathrm{x}$ & & $\mathrm{x}$ & $\mathrm{x}$ & & 6 & Excelente & $\begin{array}{l}\text { Didático - facilita a visualização dos fenômenos } \\
\text { elétricos e magnáticos. }\end{array}$ & $\begin{array}{l}\text { Uso no browser, sem necessidade } \\
\text { de instalação. }\end{array}$ \\
\hline & Logisim & $\mathrm{x}$ & & & & & $\mathbf{x}$ & $\mathbf{x}$ & & 1 & Excelente & Simplificação de circuitos lógicos. & \begin{tabular}{|l|} 
Simplicidade e facilidade de uso. \\
\end{tabular} \\
\hline & CadeSimu & & & & & & $\mathrm{x}$ & $x$ & $\mathrm{x}$ & 2 & Excelente & $\begin{array}{l}\text { O site oferece um curso gratuito, manuais e } \\
\text { fóruns de discussão. }\end{array}$ & $\begin{array}{l}\text { Grande quatidade de exemplos } \\
\text { prontos para simulação. }\end{array}$ \\
\hline & WEG CLIC02 & & & & & $\mathrm{x}$ & $\mathrm{x}$ & $\mathrm{x}$ & $\mathrm{x}$ & 1 & Excelente & Linguagens de programação (LD/FBD) & $\begin{array}{c}\text { Simulador leve, gratuito e em pt- } \\
B R \text {. }\end{array}$ \\
\hline & EKTS & & & & & & & $\mathrm{x}$ & & 1 & Satisfatório & $\begin{array}{c}\text { O simulador não calcula o fluxo de corrente e a } \\
\text { distribuição de energia nos elementos do } \\
\text { circuito, por isso, não garante os mesmos } \\
\text { resultados no ambiente real. }\end{array}$ & $\begin{array}{l}\text { Localizador de erro que indica o } \\
\text { local no qual se encontra o } \\
\text { problema do circuito. }\end{array}$ \\
\hline & Delta ISPSoft & & & & & & & $\mathrm{x}$ & & 1 & Excelente & $\begin{array}{c}5 \text { linguagens de programação (LD / FBD / SFC / } \\
\qquad \mathrm{L} / \mathrm{ST})\end{array}$ & $\begin{array}{l}\text { Permite dividir o programa em } \\
\text { páginas/partes (pou). }\end{array}$ \\
\hline & Delta WPLSoft & & & & & & & $\mathrm{x}$ & & 1 & Deficiente & Interface simples. & $\begin{array}{l}\text { Não oferece suporte a todos os } \\
\text { CLPs da série DVP. }\end{array}$ \\
\hline & PicSimLab & & & & & $x$ & & & $\mathrm{x}$ & 1 & Excelente & $\begin{array}{l}\text { São necessários dowlonds de compiladores e } \\
\text { bibliotecas }\end{array}$ & Integração com Arduino. \\
\hline & MotorAnalysis & & & & & & & $\mathrm{x}$ & & 2 & Deficiente & $\begin{array}{l}\text { Gera gráficos com valores de corrente, tensão } \\
\text { etc. }\end{array}$ & $\begin{array}{c}\text { Integração com o MatLab. Permite } \\
\text { o design de máquinas, além da } \\
\text { análise. }\end{array}$ \\
\hline & OMNeT++ & & & & & $\mathrm{x}$ & & & & 1 & Satisfatório & $\begin{array}{l}\text { Extensōes que permitem grande variedade de } \\
\text { simulaçoes. }\end{array}$ & $\begin{array}{l}\text { Criação de módulos que podem } \\
\text { ser reutilizados. }\end{array}$ \\
\hline 产 & ns-3 & $\mathrm{x}$ & & & & $\mathrm{x}$ & & $\mathrm{x}$ & & 1 & Deficiente & Interface didática. & \begin{tabular}{|c|}
$\begin{array}{c}\text { Possui modelos para redes } \\
\text { baseadas em IP ou não. }\end{array}$ \\
\end{tabular} \\
\hline 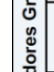 & Scada BR & $\mathrm{x}$ & & & & & $\mathrm{x}$ & & $\mathrm{x}$ & 1 & Excelente & Visualização de dados em tempo real. & $\begin{array}{l}\text { Registro de variáveis em banco de } \\
\text { dados. Gera relatório de alarmes, } \\
\text { eventos e variáveis. }\end{array}$ \\
\hline$\frac{\frac{\pi}{\vec{E}}}{\omega}$ & JMCAD & $\mathrm{x}$ & & & & & & & & 3 & Deficiente & $\begin{array}{l}\text { Usa blocos para simular sistemas dinâmicos, } \\
\text { inspirado no Simulink. }\end{array}$ & $\begin{array}{l}\text { Permite que o usuário crie blocos } \\
\text { customizados e os adicione à } \\
\text { biblioteca. }\end{array}$ \\
\hline & Qucs & $\mathrm{x}$ & & & & & & $\mathrm{x}$ & & 3 & Satisfatório & Interface simples (crua). & \begin{tabular}{|c|}
$\begin{array}{c}\text { Variedade de modelos. Ainda não } \\
\text { totalmente desenvolvido para } \\
\text { Windows. }\end{array}$ \\
\end{tabular} \\
\hline & Simullde & & & & & & & $\mathrm{x}$ & $\mathrm{x}$ & 3 & Satisfarório & $\begin{array}{c}\text { O site do simulador tem tutoriais e fórum em } \\
\text { inglês. }\end{array}$ & Interface simples e didática. \\
\hline & NGSpice & $\mathrm{x}$ & & & & & & $\mathrm{x}$ & & 4 & Excelente & $\begin{array}{c}\text { Grande diversidade de modelos para } \\
\text { componentes eletrônicos. }\end{array}$ & $\begin{array}{l}\text { Não aceita esquemáticos como } \\
\text { entrada, apenas código. }\end{array}$ \\
\hline & Timing Editor & & & & & $\mathrm{x}$ & & & & 1 & Deficiente & $\begin{array}{l}\text { Facilita a visualizacăa do funcionamento de } \\
\text { circuitos sequenciais. Possui retas verticais } \\
\text { para mediçăo de valores. }\end{array}$ & $\begin{array}{l}\text { Exporta os diagramas para } \\
\text { formatos de imagem (png). }\end{array}$ \\
\hline & Digital Logic Design & & & & & & & & & 1 & Deficiente & Interface limpa e organizada, didática. & $\begin{array}{c}\text { Converte partes de um circuito em } \\
\text { módulos, que podem ser } \\
\text { reutilizados. }\end{array}$ \\
\hline & GPSim & & & & & & & $x$ & & 1 & Deficiente & Interface que imita conexōes em HW. & Boa velocidade de simulaçăo. \\
\hline & $\begin{array}{l}\text { Electric VLSI Design } \\
\text { System }\end{array}$ & & & & & & & $\mathrm{x}$ & & 1 & Satisfatório & Há um manual em inglês do desenvolvedor. & $\begin{array}{l}\text { Checagem de consistência da } \\
\text { rede (conexäo) e essforco lógico. } \\
\text { Diversos algoritmos de } \\
\text { roteamento estáa incluidos no } \\
\text { simulador. }\end{array}$ \\
\hline & Circuit Lab & & $\mathrm{x}$ & & $\mathrm{x}$ & $x$ & & $\mathrm{x}$ & & 5 & Exelente & Plataforma intuitiva, interface simples e limpa. & $\begin{array}{c}\text { Ferramentas que aumentam a } \\
\text { produtividade coneōes fáceis de } \\
\text { fazer, aceita copy/paste de } \\
\text { componentes. }\end{array}$ \\
\hline & NanoCad & & & & & & & $x$ & & 2 & Excelente & $\begin{array}{l}\text { Opção gratuita que substitui bem } \mathrm{OAutoCad} \\
\text { (para fins didáticos) }\end{array}$ & $\begin{array}{c}\begin{array}{c}\text { Simulador leve e com interface } \\
\text { simples. }\end{array} \\
\end{array}$ \\
\hline 氩 & Matlab & & & & $\mathrm{x}$ & $\mathrm{x}$ & & $\mathrm{x}$ & $\mathrm{x}$ & 12 & Satisfatório & $\begin{array}{l}\begin{array}{c}\text { Pode ser ultilizado em várias componentes } \\
\text { (diversas toolboxes). }\end{array} \\
\end{array}$ & $\begin{array}{l}\text { Linguagem de programação fácil e } \\
\text { intuitiva. }\end{array}$ \\
\hline 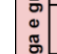 & Orcad & & & & & $\mathrm{x}$ & & $\mathrm{x}$ & & 5 & Satisfatório & $\begin{array}{l}\text { A versão gratuita limita o tamanho e a } \\
\text { complexidade do projeto }\end{array}$ & $\begin{array}{l}\text { Simulaçăoes ao longo do tempo - } \\
\text { dinâmicas. }\end{array}$ \\
\hline 年 & EveryCircuit & & $\mathrm{x}$ & $\mathrm{x}$ & $\mathrm{x}$ & $\mathrm{x}$ & $\mathrm{x}$ & $\mathbf{x}$ & & 6 & Excelente & $\begin{array}{l}\text { Interface gráfica que facilita a visualização dos } \\
\text { fenômenos elétricos e magnéticos. }\end{array}$ & $\begin{array}{c}\text { Na versão gratuita, há limite de } \\
\text { componentes }\end{array}$ \\
\hline 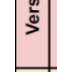 & Pycharm & & $\mathrm{x}$ & & & $x$ & & & & 11 & Satisfatório & Gratuito na versão community. & $\begin{array}{c}\text { Interface gráfica bem organizada, } \\
\text { o que facilita a programação e a } \\
\text { depuraçẫo de erros. }\end{array}$ \\
\hline & Fritzing & & & & & & $\mathrm{x}$ & $\mathrm{x}$ & $\mathrm{x}$ & 5 & Excelente & $\begin{array}{l}\text { Interface didática que emula os componentes } \\
\text { fisicos. }\end{array}$ & $\begin{array}{l}\text { Site do desenvolvedor disponiliza } \\
\text { materiais sobre a utilização do } \\
\text { simulador. }\end{array}$ \\
\hline & Protheus & & & & & $\mathrm{x}$ & & $\mathrm{x}$ & $\mathrm{x}$ & 5 & Satisfatório & $\begin{array}{l}\text { EDA integrado ao simulador (esquemático, } \\
\text { placa, renderiza a placa e simula). }\end{array}$ & $\begin{array}{l}\text { Sistema modular, possibilidade de } \\
\text { aquisiçẫo de módulos individuais } \\
\text { atualizados. }\end{array}$ \\
\hline 要 & Autocad & & & & & $\mathrm{x}$ & & $\mathrm{x}$ & $\mathrm{x}$ & 4 & Excelente & $\begin{array}{l}\text { Aplicativos e extensōes que facilitam a } \\
\text { organizaçăo dos projetos. }\end{array}$ & $\begin{array}{c}\text { Possui aplicativo para } \\
\text { dispositivos móveis, salva } \\
\text { projetos na nuvem. }\end{array}$ \\
\hline 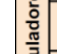 & Pycharm Professional & & & & & $\mathrm{x}$ & & $\mathrm{x}$ & & 11 & Satisfatório & Gratuito na versão community. & $\begin{array}{l}\text { Detecção o de código duplicado, } \\
\text { diagramas em UML. }\end{array}$ \\
\hline है & SimulaVR & & & & & & & $x$ & $x$ & 1 & Deficiente & Interface Python. & Possui depurador de erros. \\
\hline & Draftsight & & & & & & $\mathrm{x}$ & $x$ & $\mathrm{x}$ & 1 & Satisfatório & Integrado com Autocad e Solidworks. & $\begin{array}{l}\text { Aceita fórmulas em tabelas, } \\
\text { converte imagens em jpeg, png, } \\
\text { etc. para dwg (através de } \\
\text { rastreamento). }\end{array}$ \\
\hline & Automation Studio & & & & & $\mathrm{x}$ & $\mathrm{x}$ & $\mathrm{x}$ & $\mathrm{x}$ & 1 & Satisfatório & $\begin{array}{l}\text { Interface gráfica que facilita a visualização das } \\
\text { caracteristicas dos componentes. }\end{array}$ & $\begin{array}{l}\text { Grande quantidade de ensaios } \\
\text { podem ser simulados. }\end{array}$ \\
\hline
\end{tabular}

Fonte: elaborada pelos autores. 


\section{CONSIDERAÇÕES FINAIS}

O presente trabalho apresenta como objetivo a elaboração de um quadro comparativo que não apenas sugira simuladores para componentes curriculares, mas também os classifica segundo parâmetros comparativos. Dez critérios foram estabelecidos na busca de qualificação dos simuladores avaliados segundo custo, acessibilidade, plataforma de hardware necessária, usabilidade e praticidade. São eles: (1) ser Open Source, (2) possuir aplicativo para Android, (3) possuir aplicativo para iOS, (4) possibilitar uso no browser, (5) ter sido atualizado nos últimos 2 anos, (6) possuir versão em língua portuguesa, (7) realizar simulações através de esquemáticos, (8) realizar simulações que emulam a montagem física, (9) número de disciplinas atendidas e (10) documentação disponível.

O escopo deste trabalho envolve as componentes curriculares específicas da área técnica nos cursos de Engenharia Elétrica e técnico em Automação Industrial do IFPR campus Campo Largo. No entanto, como pode ficar evidente nos resultados apresentados, as disciplinas avaliadas são comuns a cursos da área de Elétrica. Espera-se, portanto, que as análises feitas possam auxiliar docentes e discentes de diferentes instituições. Neste trabalho foram considerados 28 componentes curriculares e 35 simuladores. Através dos critérios pré-estabelecidos, chegou-se a uma relação entre os softwares disponíveis e suas possibilidades de aplicação em diferentes componentes.

Diante do quadro comparativo obtido, espera-se que docentes e discentes possam obter informações consolidadas, visando aumentar as chances de que uma boa escolha de ferramenta de simulação seja feita de acordo com cada situação de uso. Entende-se que as informações reunidas também podem ser úteis na tomada de decisão de gestores, que em alguns casos precisam escolher e especificar licenças de softwares a serem adquiridas.

Como trabalhos futuros, espera-se realizar comparativos entre resultados obtidos de montagens físicas e de simulações. Estas comparações permitirão a comparação das experiências de estudantes com montagens físicas e ferramentas computacionais.

\section{REFERÊNCIAS}

ALSADOON, A.; PRASAD, P. W. C.; BERG, A. Using software simulators to enhance the learning of digital logic design for the information technology students. European Journal of Engineering Education, v. 42, n. 5, p. 533-546, 2016.

AYASUN, S.; NWANKPA, C. Induction motor tests using Matlab/Simulink and their integration into undergraduate electric machinery courses. IEEE Transactions on Education, v. 48, n. 1, p. 37-45, 2005.

BERRY, F.C.; DIPIAZZA, P.S.; SAUER, S. L. The future of electrical and computer engineering education. IEEE Transactions on Education, v. 14, n. 4, p. 467-476, 2003.

CAMPBELL et al. The effectiveness of learning simulations for electronic laboratories. Journal of Engineering Education, v. 91, n. 1, p. 81-87, 2002.

CAÑIZARES, C. A.; FAUR, Z. T. Advantages and disadvantages of using various computer tools in electrical engineering courses. IEEE Transactions on Education, v. 40, n. 3, p. 166-171, 1997. 
FELDER, R. M. et al. The future of engineering education II. Teaching methods that work. Chemical Engineering Education, v. 34, n. 1, p. 26-39, 2000.

HERON, M.; HANSON, V. L.; RICKETTS, I. Open source and accessibility: advantages and limitations. Journal of Interaction Science, v. 1, n. 2, p. 1-10, 2013.

JUUSO, E. K.; SORSA, A. A new teaching scheme for advanced control courses. IFAC Proceedings Volumes, v. 24, n. 24, p. 49-54, 2010.

LORETO-GÓMEZ, G. et al. Analysing the effect of the use of 3D simulations on the performance of engineering students in a robotics curse: finding from a pilot study. International Journal of Electrical Engineering and Education, v. 56, n. 2, p. 163-178, 2019.

RUGARCIA, A. et al. The future of engineering education I. A vision for a new century. Chemical Engineering Education, v. 36, n. 1, p. 16-25, 2000.

SÁ, M. J; SERPA, S. The Global Crisis Brought about by SARS-CoV-2 and Its Impacts on Education: An Overview of the Portuguese Panorama. Science Insight Education, v. 5, n. 2, p. 525-530, 2020.

SWAIN, N. K.; ANDERSON, J. A. Role of simulation software in enhancing student learning in computer organization and microcontroller curses. In: IAJC-IJME International Conference, 2008, Nashville, TN, EUA. Disponível em: https://www.ijme.us/cd 08/PDF/135\%20ENT\%20207.pdf. Acesso em: 19/04/2021.

\title{
COMPARISON OF SIMULATORS ON ELECTRICAL ENGINEERING AND INDUSTRIAL AUTOMATION WITH FOCUS ON REMOTE LEARNING
}

\begin{abstract}
The COVID-19 pandemic imposed a series of difficulties on society. Among these, the need to teach and learn remotely and urgently. The remote teaching modality is especially challenging in highly technological courses, in which experimentation is fundamental for a better understanding of the concepts. In Electrical Engineering and its ramifications, which demand the understanding of several abstract phenomena, laboratories are of particular importance. Simulators have great potential to assist both teachers and students in the teaching-learning process. However, given the wide range of simulators available, choosing one that fits well in each situation may not be a simple task. Therefore, this work proposes the elaboration of a comparative table of simulators available for technical disciplines in the areas of Electrical Engineering and Industrial Automation, listing some of its advantages and disadvantages. The simulators were listed and evaluated according to a series of criteria related to cost, accessibility, necessary hardware platform, usability and practicality. 28 curriculum components were evaluated, for which it was possible to suggest some of the 30 evaluated simulation tools. The results obtained so far are presented in the form of tables that summarize important characteristics and aim to facilitate choices and uses by students, teachers and managers of educational institutions.
\end{abstract}

Keywords: Simulators; Electrical Engineering Education; Remote Learning; Computer Tools. 